\title{
A Geometric Realization Without Self-Intersections Does Exist for Dyck's Regular Map
}

\author{
Jürgen Bokowski \\ Technische Hochschule Darmstadt, Fachbereich Mathematik, Schlossgartenstrasse 7, \\ 6100 Darmstadt, Federal Republic of Germany
}

\begin{abstract}
Even in our decade there is still an extensive search for analogues of the Platonic solids. In a recent paper Schulte and Wills [13] discussed properties of Dyck's regular map of genus 3 and gave polyhedral realizations for it allowing self-intersections. This paper disproves their conjecture in showing that there is a geometric polyhedral realization (without self-intersections) of Dyck's regular map $\{3,8\}_{6}$ already in Euclidean 3-space. We describe the shape of this new regular polyhedron.
\end{abstract}

\section{Introduction}

One approach to looking for analogues of the Platonic solids is to determine those combinatorial 2-manifolds without boundary, of given genus $g$, which admit a flag-transitive group of automorphisms, and then to find suitable polyhedral realizations, i.e. (in the case of Euclidean 3-space $E^{3}$ ), a finite set of plane polygons whose union (without self-intersections) corresponds to the combinatorial manifold.

There is a classical theory to determine such combinatorial manifolds (regular maps). They are known up to genus 6 , and some of them are famous examples, such as Dyck's map $\{3,8\}_{6}$ and Klein's $\{3,7\}_{8}$ of genus 3 .

An answer to the question how to find in general suitable polyhedral realizations is unknown. For Klein's regular map $\{3,7\}_{8}$ a realization was found by Schulte and Wills [12] and they conjectured in another paper [13] that there is no realization for Dyck's map in $E^{3}$.

In this paper we disprove their conjecture [13, p. 142], in showing that there is a geometric realization (without self-intersections) for Dyck's regular map already in Euclidean 3-space. 


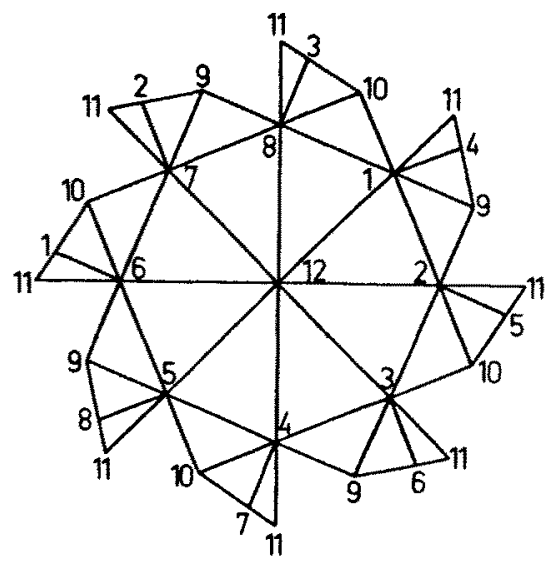

Fig. 1

We would like to refer the reader to the paper of Schulte and Wills [13], and the papers cited there, for more details about the maps and the context in which they were discussed.

In Section 4 we illustrate the shape of our realization as far as projections allow this attempt. A better understanding is given in a forthcoming computeraided film about Dyck's map.

\section{Description of Dyck's Map $\{3,8\}_{6}$}

For our purposes a very short description of Dyck's map $\{3,8\}_{6}$ will be sufficient. Compare the original papers of Dyck from 1880 [6], [7], [8].

Figure 1 shows the (oriented) combinatorial manifold (without boundary). Equally labeled vertices have to be identified. Note that the labeling is not the same as in [13].

As the map has $f_{0}=12$ vertices, $f_{1}=28$ edges, and $f_{2}=32$ triangular faces, Euler's formula

$$
f_{0}-f_{1}+f_{2}=2-2 g
$$

implies that it is of genus $\mathbf{g}=3$. Its flag-transitive automorphism group has order 192. Perhaps this high symmetry is better understood by looking at its representation by an algebraic curve of order 4 with homogeneous complex variables $x, y$, and $z$, namely

$$
x^{4}+y^{4}+z^{4}=0
$$

\section{The Geometric Realization}

The author's method of deciding special geometrical realization problems, which was successful in the past (see [1]-[3] and [5]), and which was first described 
in more detail together with further properties in a joint paper with Sturmfels [4], was first applied to this problem, too. Finally, the author was convinced that because of the combinatorial structure there would be no hope to find a corresponding matroid manifold (or chirotope manifold) and stopped the timeconsuming computational investigations.

The main difficulty is given by the structure of the links of the four vertices 9, 10, 11 and 12 (see Fig. 2). To avoid one twisted situation in one of these pictures seems to cause new twistings in others.

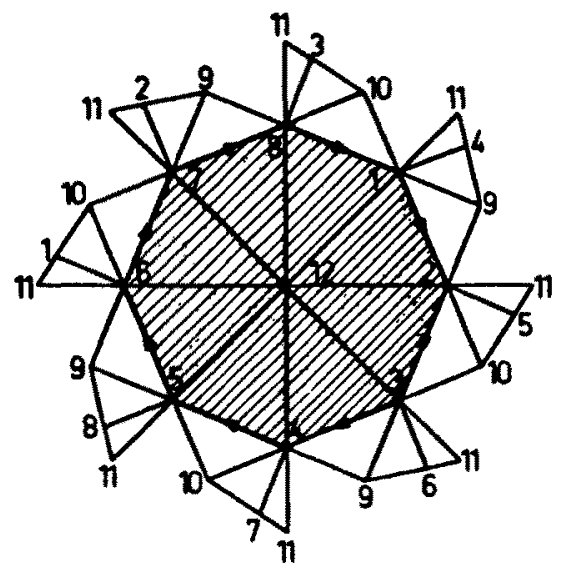

star of 12

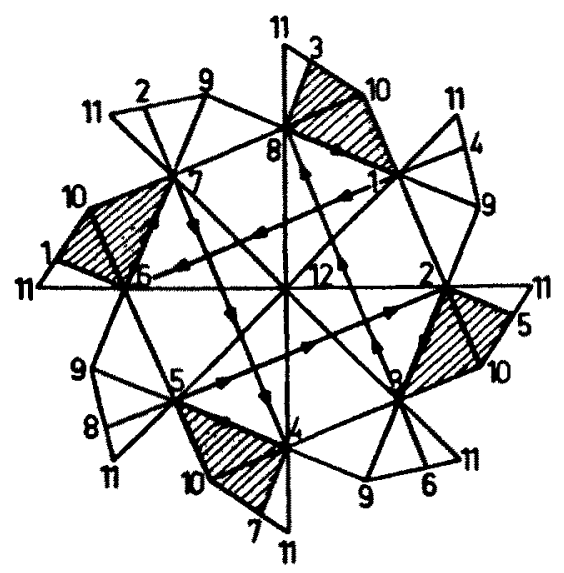

star of 10

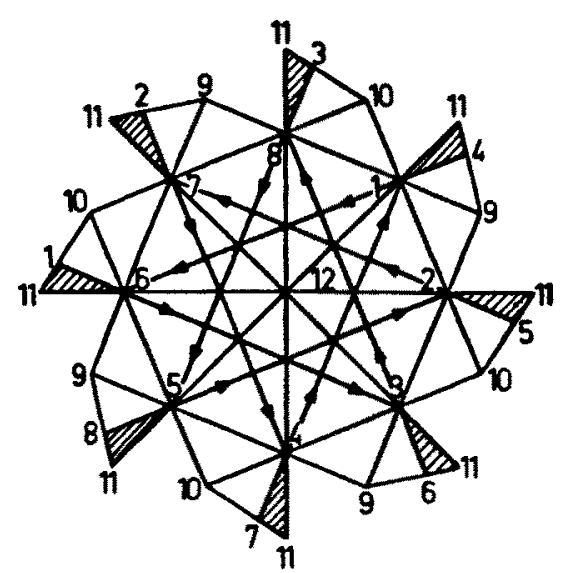

star of 11

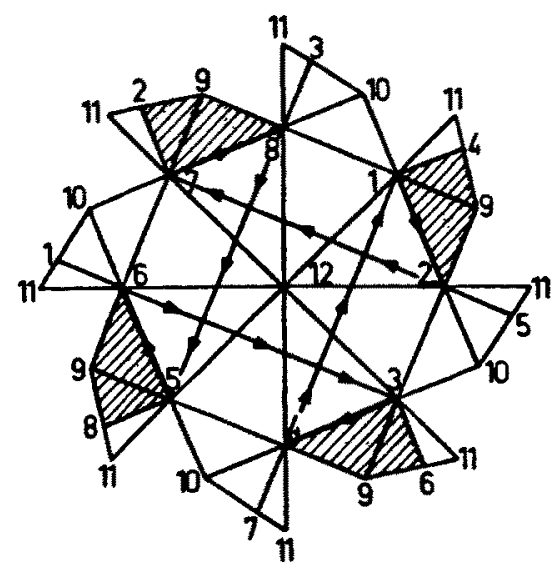

star of 9

Fig. 2 
After looking for a proof for nonrealizability we found, after all, by intuition, the following coordinates of a realization:

\begin{tabular}{|c|c|c|c|}
\hline 1 & 0.707 & 0.707 & 0 \\
\hline 2 & 1 & 0 & 0 \\
\hline 3 & 0.707 & -0.707 & 0 \\
\hline 4 & 0 & -1 & 1 \\
\hline 5 & -0.707 & -0.707 & -2 \\
\hline 6 & -1 & 0 & 0 \\
\hline 7 & -0.707 & 0.707 & 2 \\
\hline 8 & 0 & 1 & -1 \\
\hline 9 & -0.636 & 0.636 & 1 \\
\hline 10 & -0.636 & -0.636 & -1 \\
\hline 11 & 0.9 & 0 & 0 \\
\hline 12 & 10 & 0 & -100 \\
\hline
\end{tabular}

Therefore we have the following:

Theorem 1. A geometric realization (without self-intersections and with flat triangles) for Dyck's regular map $\{3,8\}_{6}$ on a surface of genus 3 does exist in Euclidean 3-space.

Proof. After obtaining the coordinates (see above) and the list of triangles and edges it is, in principle, now easy to check that no edge intersects any triangle. But, of course, this takes some time, and, also, the shape of the realization is not seen. Therefore the next section gives a more comprehensive understanding of the geometric situation.

\section{The Shape of the Polyhedron for Dyck's Map $\{3,8\}_{6}$}

In this section we describe the shape of the polyhedron for Dyck's map $\{3,8\}_{6}$. The orthogonal projection onto the $x y$-plane of the link of point 12 is a regular octagon with center 0 , and all further projected points lie in the interior of this octagon. Thus there is no problem in realizing the star of point 12 if point 12 is suitably chosen as $(0,0, z)$ with a very small $z$. In our discussion we can omit these eight triangles containing 12 as a vertex in order to get a better understanding of the rest of the polyhedron.

We have drawn orthogonal projections onto the $x y$-plane of the stars of the points 11,9 , and 10 in Figs. 3, 4, and 5, respectively. The 24 triangles of these stars admit a half-turn about the $x$-axis as a symmetry; it keeps the star of 11 fixed and maps the star of 9 onto the star of 10 and vice versa. However, this 


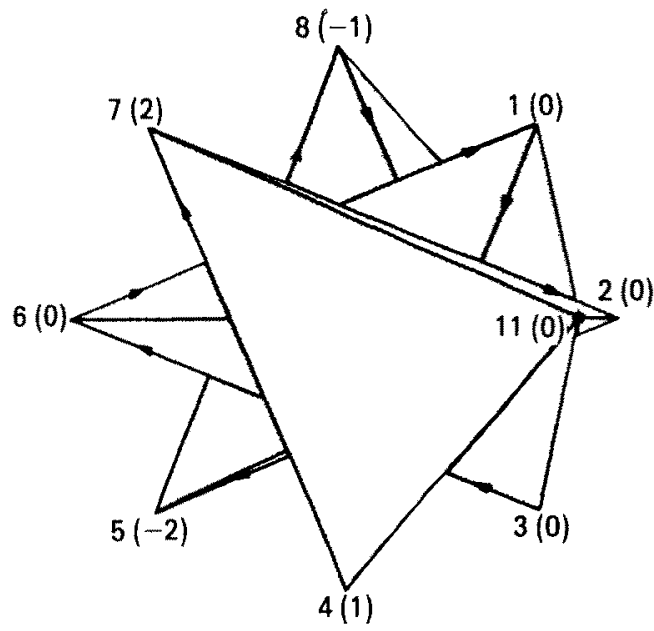

Fig. 3

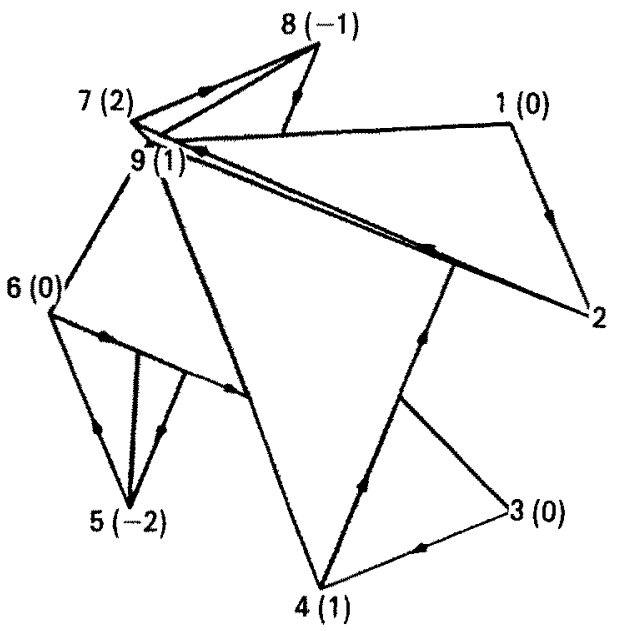

Fig. 4

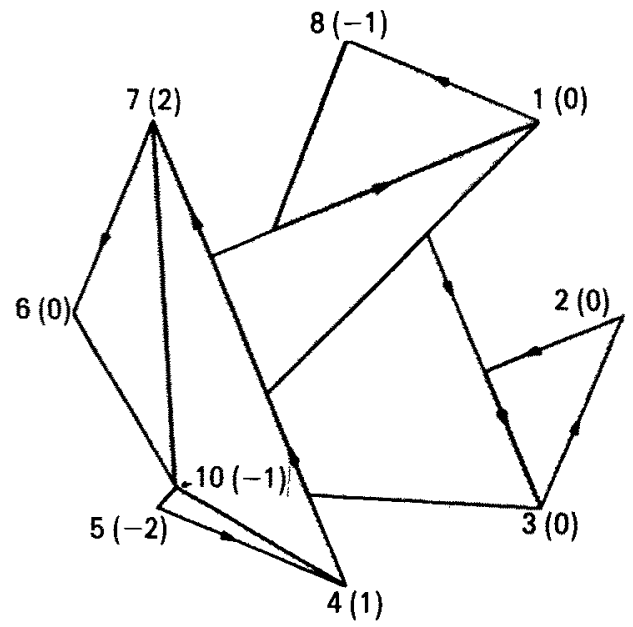

Fig. 5

half-turn will not be a symmetry of the whole polyhedron. In fact, because of the choice of vertex 12 the polyhedron will not have any Euclidean symmetry at all.

In the following figures the symbols $8(-1)$, etc., are used to denote the vertex 8 (with $z$-coordinate -1 ). The vertices are assumed to be arranged in the same way as in Fig. 2.

It is very easily seen from the projections, together with the z-coordinates, that each star has no self-intersections. But do all stars altogether have no self-intersections, too? We try to demonstrate this for any pair of stars. Because 


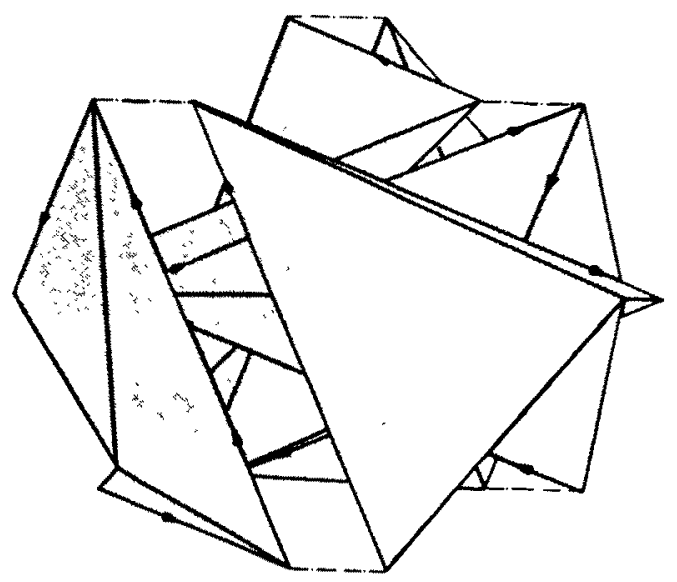

Fig. 6

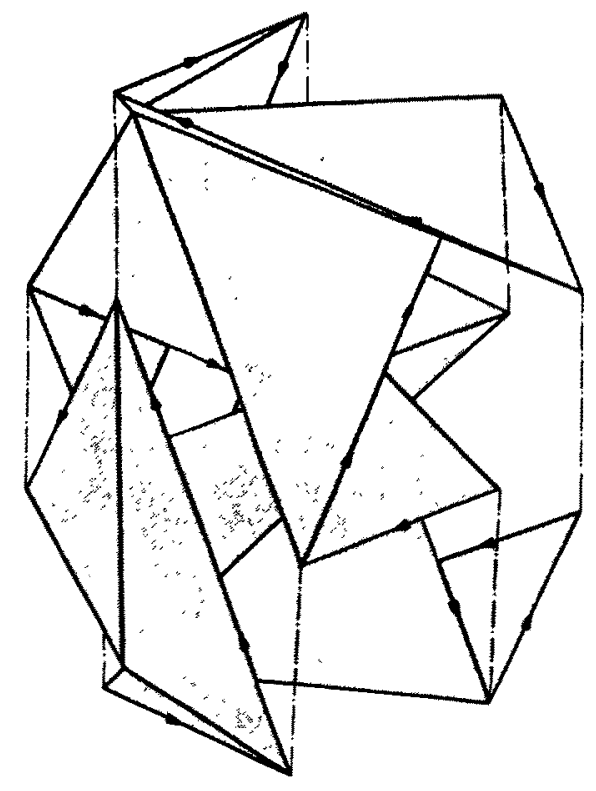

Flg. 7

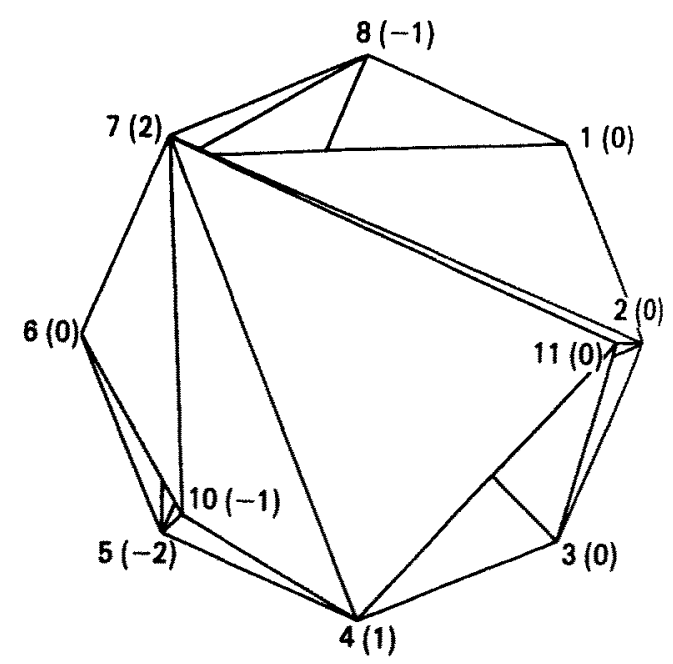

Fig. 8

of the above-described symmetry we have to deal only with two pairs of stars.

Figure 6 shows how to move the star of 9 or 10 horiozontally to the star of 11 in order to join both. Figure 7 shows the situation for the other pairs of stars. A complete view of our model is seen in Fig. 8.

We would like to mention again that a better understanding of the geometric situation is given in a forthcoming computer-aided film. 


\section{Final Remarks}

The list of regular maps of genus at most 6 is known, complete classifications can be found in Sherk [16] and Garbe [9]. But the question of whether, in all these cases, a corresponding geometric polyhedral realization exists is still open.

In each case the question of the highest symmetry of the realization, as well as the corresponding question for the dual maps, is very natural.

\section{References}

1. C. Antonin, Ein Algorithmusansatz für Realisierungsfragen im $E^{d}$ getestet an kombinatorischen 3-Sphären, Staatsexamensarbeit, Bochum, 1982.

2. J. Bokowski and K. Garms, Altshuler's sphere $M_{425}^{10}$ is not polytopal, European J. Combin., to appear.

3. J. Bokowski and 1. Shemer, Neighborly 6-polytopes with 10 vertices, Israel J. Math. 58 (1987), 103-124.

4. 3. Bokowski and B. Sturmfels, On the coordinatization of oriented matroids, Discrete Comput. Geom. 1 (1986), 293-306.

5. J. Bokowski, G. Ewald, and P. Kleinschmidt, On combinatorial and affine automorphisms of polytopes, Israel J. Math. 47 (1984), 123-130.

6. W. Dyck, Uber Aufstellung und Untersuchung von Gruppe und Irrationalität regulärer Riemannscher Flächen, Math. Ann. 17 (1880), 473-508.

7. W. Dyck, Notiz über eine reguläre Riemannsche Fläche vom Geschlecht 3 und die zugehörige Normalkuve 4. Ordnung, Math. Ann. 17 (1880), 510-516.

8. W. Dyck, Gruppentheoretische Studien, Math. Ann. 20 (1882), 1-45.

9. D. Garbe, Über die regulären Zerlegungen geschlossener Flächen, J. Reine Angew. Math. 237 (1969) 39-55.

10. P. McMullen, C. Schulz, and J. M. Wills, Equivelar polyhedral manifolds in $E^{3}$, Israel J. Math. 41 (1982), 331-346.

11. P. McMullen, E. Schulte, and J. M. Wills, Infinite series of combinatorially regular polyhedra in three-space, in preparation.

12. E. Schulte and J. M. Wills, A polyhedral realization of Felix Klein's map $\{3,7\}_{8}$ on a Riemannian manifold of genus 3, J. London Math. Soc. 32 (1985), 539-547.

13. E. Schulte and J. M. Wills, Geometric realizations for Dyck's regular map on a surface of genus 3, Discrete Comput. Geom, 1 (1986), 141-153.

14. E. Schulte and J. M. Wills, On Coxeter's regular skew polyhedra, Discrete Math.60 (1986), 253-262.

15. E. Schulte and J. M. Wills, Combinatorially Regular Polyhedra in Three-Space, Preprint, Siegen, Vol. 179, 1986.

16. F. A. Sherk, The regular maps on a surface of genus 3, Canad. J. Math. 11 (1959), 452-480.

Received November 3, 1986, and in revised form April 29, 1987. 